\title{
On the Q-Exponential Type Boltzmann Factors from the Super-Statistics and the Q-Deformed Maxwell Boltzmann Distribution
}

\author{
Won Sang Chung* \\ Department of Physics and Research Institute of Natural Science, \\ College of Natural Science, Gyeongsang National University, Jinju 660-701, Korea \\ E-mail : *mimip4444@ hanmail.net
}

Received 31 December 2015, Accepted 16 March 2016

\begin{abstract}
In this paper we use the super-statistics theory to obtain three q-exponential type Boltzmann factors. We apply three types of effective Boltzmann factors to the kinetic theory of an ideal gas. We also compute the most probable speeds, average speeds and averages of squared velocity and discuss the q-deformed equipartition theorem for three types Boltzmann factors.
\end{abstract}

Keywords: Super-statistics; effective Boltzmann factor; non-extensive entropy; Boltzmann factor; Tsallis entropy; Maxwell-Boltzmann distribution;, equipartition theorem.

\section{Introduction}

Super-statistics is a superposition of two or more different statistics: One given by ordinary Boltzmann factors, and another one given by the fluctuations of the intensive parameter such as the inverse temperature. Nonlinear dynamical processes often create a fluctuating environment for a given mesoscopic system [1]. As an effective theory for this complex system, the super-statistics concept arose. In this direction, much work has been accomplished for further theoretical elaboration [2-10].

In 2003, the super-statistics was first introduced by Beck and Cohen [1]. In the equilibrium thermodynamic system, the Boltzmann factor $e^{-\beta E}$ plays an important role for computing the macroscopic quantities such as the entropy, internal energy and Helmholtz free energy. Here $\beta$ is regarded as an inverse temperature $=1 / k_{B} T$, where $k_{B}$ is a Boltzmann constant. However, in the non-equilibrium thermodynamical system, Boltzmann factor dose not preserve its shape in the long time evolution any more. This means that $\beta$ may fluctuate around $1 / k_{B} T$, so it is not an inverse temperature but a random variable. Thus, in this system, we need the effective Boltzmann factor which is an average of the ordinary Boltzmann factor.

The effective Boltzmann factor $\mathrm{B}(\mathrm{E})$ is defined as

$\mathrm{B}(\mathrm{E})=\int_{0}^{\infty} e^{-\beta^{\prime} E} f\left(\beta^{\prime}, \beta\right) d \beta^{\prime}$

where $f\left(\beta^{\prime}, \beta\right)$ is an probability density obeying

$\int_{0}^{\infty} f\left(\beta^{\prime}, \beta\right) d \beta^{\prime}=1$

There has been a growing interest in generalizing the Boltzmann-Gibbs statistical mechanics. Because the entropy plays a fundamental role in the statistical physics, the entropy should be deformed so as to construct a new (deformed) theory. The first attempt has been accomplished by Tsallis $[11,12]$. Based on the fact that Boltzmann-Gibbs theory is not adequate for various complex, natural, artificial and social system, he introduced the non-extensive entropy is given by

$$
S_{q}=k\left(\sum_{i}^{W} p_{i}^{1-q}-1\right) / q,(q>0) \text {. The non-extensive }
$$
Boltzmann-Gibbs entropy has attracted much interest among mathematicians and physicists who study thermodynamics of complex system [13-15]. When the deformation parameter $q$ goes to 1 , the non-extensive entropy reduces to the ordinary one. Tsallis entropy can be written as

$S_{q}=-k \sum_{i=1}^{W} p_{i}^{1-q} \ln _{q} p_{i}=k \sum_{i=1}^{W} p_{i} \ln _{q} \frac{1}{p_{i}}$

where $W$ is a number of microstates and the $q$ logarithmic function is defined as

$\ln _{q} t=\left\{\begin{array}{c}\frac{x^{q}-1}{q}, \quad(x>0, q \neq 0) \\ \ln x \quad(q=0)\end{array}\right.$

and its inverse is given by

$\mathrm{e}_{q}(x)=\left\{\begin{array}{cl}(1+q x)^{1 / q}, & (x, q \in R, q \neq 0) \\ e^{x} & (q=0)\end{array}\right.$

This entropy gives the following probability distribution:

$p_{i}=\frac{1}{Z_{q}}\left[e_{q}\left(-\beta E_{i}\right)\right]^{-1}$

where

$Z_{q}=\sum_{i}^{W}\left[e_{q}\left(-\beta E_{i}\right)\right]^{-1}$ 
Here we have a question: Is there any other choice of the deformed exponential? We can say yes. For example, we can introduce the following two cases instead of the Eq.(5):

$p_{i}=\frac{1}{Z_{q}}\left[e_{q}\left(-\beta E_{i}\right)\right]^{\beta}$

$p_{i}=\frac{1}{z_{q}}\left[e_{q}(-\beta)\right]^{E_{i}}$

Now we will denote the probabilities given in the Eq.(6), Eq.(8) and the Eq.(9) by Type I , Type II and Type III, respectively. In this paper we use the super-statistics theory to obtain three $q$-exponential type Boltzmann factors. We apply three types of effective Boltzmann factors to the kinetic theory of an ideal gas. We also compute the most probable speeds, average speeds and averages of squared velocity and discuss the $q$-deformed equipartition theorem for three types Boltzmann factors.

\section{Three q-Exponential Type Boltzmann Factors}

In this section we choose suitable probability distributions $\beta$ to obtain three $q$-exponential type Boltzmann factors designated by Type I, II and III. For the choice of $f\left(\beta^{\prime}, \beta\right)=\delta\left(\beta^{\prime}-\beta\right)$, we have

$\mathrm{B}(\mathrm{E})=e^{-\beta E}$

However, different choices of $f\left(\beta^{\prime}, \beta\right)$ yield various effective Boltzmann factor $\mathrm{B}(\mathrm{E})$. In the following subsections we discuss some choices of $f\left(\beta^{\prime}, \beta\right)$ which give three types of Boltzmann factors.

\subsection{Type I}

Now let us consider the following distribution:

$\mathrm{f}(\beta)=C e^{-a \beta} \beta^{b}$

where the normalization constant $C$ is given by:

$C^{-1}=a^{-1-b} \Gamma(b+1)$

and $a>0, b>0$ is assumed. The average $\langle\beta\rangle=\beta_{0}$ is defined as

$\beta_{0}=\int_{0}^{\infty} \beta f(\beta) d \beta=a^{-1}(1+b)$

and the variance $\sigma_{0}^{2}$ is

$\sigma_{0}^{2}=a^{-2}(b+1)$

Thus, we have

$\mathrm{a}=\frac{\beta_{0}}{\sigma_{0}^{2}}, \mathrm{~b}=\frac{\beta_{0}^{2}}{\sigma_{0}^{2}}-1$

For $q<1$, we can set $\mathrm{b}=-1+1 / q, a^{-1}=q \beta_{0}$, which gives an effective Boltzmann factor derived from the Tsallis entropy:

$B(E)=\left[e_{q}\left(-\beta_{0} E\right)\right]^{-1}$

Expanding the Eq. (16) for small $\beta_{0} E$, we have
$\mathrm{B}(\mathrm{E})=e^{-\beta_{0} E}\left(1-\frac{1}{2} q \beta_{0}{ }^{2} E^{2}-\frac{1}{3} q^{2} \beta_{0}{ }^{3} E^{3}+\cdots\right)$

\subsection{Type II}

If we choose $\mathrm{f}(\beta)=\delta\left(\beta-\beta_{0}\right)$, the effective Boltzmann factor reduces to the ordinary Boltzmann factor. In this case $\beta_{0}$ is regarded as an equilibrium inverse temperature. In a non-equilibrium situation we assume that the peak of the Dirac delta distribution does not appear at $\beta_{0}$ but at the slightly deformed inverse temperature $g_{q}\left(\beta_{0}\right)$, where $g_{q}\left(\beta_{0}\right)$ is an smooth function depending on the parameter q. In this case the distribution becomes

$\mathrm{f}(\beta)=\delta\left(\beta-g_{q}\left(\beta_{0}\right)\right)$

where $\mathrm{q}>0$ is assumed. The average is given by

$\langle\beta\rangle=g_{q}\left(\beta_{0}\right)$

and the variance becomes zero. The interesting choice for this distribution is

$g_{q}\left(\beta_{0}\right)=-\frac{1}{q} \ln \left(1-q \beta_{0}\right)$

where we assumed that $1-q \beta_{0} \geq 0$. Then, the effective Boltzmann factor reads

$\mathrm{B}(\mathrm{E})=\left[e_{q}(-\beta)\right]^{E}=[1-q \beta]^{E / q}$

If we demand that $1-q \beta_{0} \geq 0$, we have $T \geq T_{\text {min }}$, where $T_{\min }=q / k_{B}$ is a minimum temperature. Unless $q=0$, the minimum temperature of the system is not zero and is determined in terms of $q$.

Expanding the Eq. (21) for small $\beta_{0} E$, we have

$\mathrm{B}(\mathrm{E})=e^{-\beta_{0} E}\left(1-\frac{1}{2} q \beta_{0}{ }^{2} E-\frac{1}{3} q^{2} \beta_{0}{ }^{3} E+\cdots\right)$

\subsection{Type III}

Now let us consider the following distribution

$\mathrm{f}(\beta)=\mathrm{C} e^{-a \beta} \beta^{b}$

where the normalization constant $\mathrm{C}$ is given by

$C^{-1}=a^{-1-b} \Gamma(b+1)$

and $a>0, b>0$ is assumed. For $q>0$, we can setb $=$ $-1+\frac{\beta_{0}}{q}, a^{-1}=q$, which gives an effective Boltzmann factor derived from the Tsallis entropy:

$\mathrm{B}(\mathrm{E})=\left[e_{q}(E)\right]^{-\beta_{0}}$

Expanding the Eq. (25) for small $\beta_{0}$, we have

$\mathrm{B}(\mathrm{E})=e^{-\beta_{0} E}\left(1+\frac{1}{2} q \beta_{0} E^{2}-\frac{1}{3}\left(q^{2} \beta_{0}\right) E^{3}+\cdots\right)$

\section{Kinetic Theory of Ideal Gas}

Now let us apply three types of effective Boltzmann factors to the kinetic theory of an ideal gas. First let us consider the Maxwell-Boltzmann distribution of molecular speeds in a gas which is actually a probability density 
function of a continuous variable, $\mathrm{v}=\sqrt{v_{x}^{2}+v_{y}^{2}+v_{z}^{2}}$, the speed of a molecule. Then, the probability of finding a particle with speed $v$ is given by

$\mathrm{p}(v)=\frac{1}{Z_{v}} B\left(\frac{1}{2} m v^{2}\right) 4 \pi v^{2} d v$

where

$Z_{v}=\int_{0}^{\infty} B\left(\frac{1}{2} m v^{2}\right) 4 \pi v^{2} d v$

The most probable speed $v_{m}$ is found by finding the maximum of $(v)$ :

$\left.\frac{d}{d t} p(v)\right|_{v=v_{m}}=0$

The average speed $v_{a v}$ is defined as

$v_{a v}=\int_{0}^{\infty} v p(v) d v$

We also break the velocity $\vec{v}$ down into its components $v_{x}, v_{y}, v_{z}$. Then, the probability of finding a particle with components $v_{x}, v_{y}, v_{z}$ in the range $\mathrm{d} v_{x}, \mathrm{~d} v_{y}, \mathrm{~d} v_{z}$ is given by

$\mathrm{P}\left(v_{x}, v_{y}, v_{z}\right) \mathrm{d} v_{x}, \mathrm{~d} v_{y}, \mathrm{~d} v_{z}=\frac{1}{Z} B\left(\frac{1}{2} m v^{2}\right)$

where the effective partition function for velocity is given by

$\mathrm{Z}=\int_{-\infty}^{\infty} \int_{-\infty}^{\infty} \int_{-\infty}^{\infty} \mathrm{d} v_{x}, \mathrm{~d} v_{y}, \mathrm{~d} v_{z} B\left(\frac{1}{2} m v^{2}\right)$

The averages of squared velocity components are

$\left\langle v_{i}^{2}\right\rangle=\int_{-\infty}^{\infty} \int_{-\infty}^{\infty} \int_{-\infty}^{\infty} v_{i}^{2} \mathrm{P}\left(v_{x}, v_{y}, v_{z}\right) \mathrm{d} v_{x}, \mathrm{~d} v_{y}, \mathrm{~d} v_{z}$

\subsection{Type I}

In this case the probability of finding a particle with speed $v$ is given by

$\mathrm{p}(\mathrm{v})=4 \pi v^{2} \frac{1}{Z_{v}}\left[e_{q}\left(\frac{1}{2} \beta_{0} m v^{2}\right)\right]^{-1}$

where,

$Z_{v}=\left\{\begin{array}{c}\int_{0}^{\infty} B\left(\frac{1}{2} m v^{2}\right) 4 \pi v^{2} d v \text { if } 0<q<1 \\ \int_{0}^{\infty} B\left(\frac{1}{2} m v^{2}\right) 4 \pi v^{2} d v \text { if } q<0\end{array}\right.$

where $v_{0}=\sqrt{\frac{2}{|q| m \beta_{0}}}$. The most probable speed $v_{m}$ is given by

$v_{m}=\left\{\begin{array}{c}\sqrt{\frac{2}{(1-q) m \beta_{0}}} \text { for } 0<q<1 \\ \sqrt{\frac{2}{(1+|q|) m \beta_{0}}} \text { for } q<0\end{array}\right.$

The average speed $v_{a v}$ is then given by $v_{a v}=\left\{\begin{array}{c}\sqrt{\frac{8}{q \pi m \beta_{0}}} \frac{\Gamma(1 / q-2)}{\Gamma(1 / q-3 / 2)} \text { for } 0<q<1 \\ \sqrt{\frac{8}{|q| \pi m \beta_{0}}} \frac{\Gamma(1 /|q|+5 / 2)}{\Gamma(1 /|q|+1)} \text { for } q<0\end{array}\right.$

For $0<q<1$, the averages of squared velocity components are

$\left\langle v_{x}^{2}\right\rangle=\left\langle v_{y}^{2}\right\rangle=\left\langle v_{z}^{2}\right\rangle=\frac{2}{m \beta_{0}(2-5 q)}$

Hence,

$\left\langle\frac{1}{2} m v_{x}^{2}\right\rangle=\left\langle\frac{1}{2} m v_{y}^{2}\right\rangle=\left\langle\frac{1}{2} m v_{z}^{2}\right\rangle=\frac{k T}{2-5 q}$

which is the $q$-deformed equipartition theorem.

For $\mathrm{q}<1$, the averages of squared velocity components are

$\left\langle v_{x}^{2}\right\rangle=\left\langle v_{y}^{2}\right\rangle=\left\langle v_{z}^{2}\right\rangle=\frac{2}{m \beta_{0}(2+5|q|)}$

Hence,

$\left\langle\frac{1}{2} m v_{x}^{2}\right\rangle=\left\langle\frac{1}{2} m v_{y}^{2}\right\rangle=\left\langle\frac{1}{2} m v_{z}^{2}\right\rangle=\frac{k T}{2+5|q|}$

which is the $q$-deformed equipartition theorem.

\subsection{Type II}

In this case the probability of finding a particle with speed $v$ is given by

$\mathrm{p}(v)=4 \pi v^{2} \frac{1}{Z_{v}}\left[e_{q}\left(-\beta_{0}\right)\right]^{-\frac{1}{2} m v^{2}}$

where,

$Z_{v}=\int_{0}^{\infty} 4 \pi v^{2}\left[e_{q}\left(-\beta_{0}\right)\right]^{-\frac{1}{2} m v^{2}} d v$

The most probable speed $v_{m}$ is given by

$v_{m}=\sqrt{-\frac{2 q}{m \ln \left(1-q \beta_{0}\right)}}$

The average speed $v_{a v}$ is then given by

$v_{a v}=\sqrt{-\frac{8 q}{m \ln \left(1-q \beta_{0}\right)}}$

The averages of squared velocity components are

$\left\langle v_{x}^{2}\right\rangle=\left\langle v_{y}^{2}\right\rangle=\left\langle v_{z}^{2}\right\rangle=-\frac{q}{m \ln \left(1-q \beta_{0}\right)}$

which is the $q$-deformed equipartition theorem.

\subsection{Type III}

In this case the probability of finding a particle with speed $v$ is given by

$\mathrm{p}(v)=4 \pi v^{2} \frac{1}{Z_{v}}\left(1+\frac{1}{2} q m v^{2}\right)^{-\beta_{0} / q}$

where, 
$Z_{v}=\int_{0}^{\infty} 4 \pi v^{2}\left(1+\frac{1}{2} q m v^{2}\right)^{-\beta_{0} / q} d v$

The most probable speed $v_{m}$ is given by

$v_{m}=\sqrt{\frac{2}{m\left(1-q \beta_{0}\right)}}$

The average speed $v_{a v}$ is then given by

$v_{a v}=\sqrt{\frac{8}{q \pi m} \frac{\Gamma\left(\beta_{0} / q\right)}{\left(\beta_{0} / q-2\right)\left(\beta_{0} / q-1\right) \Gamma\left(\beta_{0} / q-3 / 2\right)}}$

The averages of squared velocity components are

$\left\langle v_{x}^{2}\right\rangle=\left\langle v_{y}^{2}\right\rangle=\left\langle v_{z}^{2}\right\rangle=-\frac{2}{m\left(2 \beta_{0}-5 q\right)}$

which is the $q$-deformed equipartition theorem.

\section{Concluding Remarks}

In this paper we used the super-statistics theory to obtain three $q$-exponential type Boltzmann factors which we call Type I, II and III. We found that Type I Boltzmann factor is well defined for $q<1$, Type II Boltzmann factor is well defined for $0<q<1 / \beta_{0}$ and Type III Boltzmann factor is well defined for $q>0$. We applied three types of effective Boltzmann factors to the kinetic theory of an ideal gas which implies that the Maxwell-Boltzmann distribution of molecular speed is q-deformed in three ways. We also computed the most probable speeds, average speeds and averages of squared velocity for three types Boltzmann factors. Finally we reached the $q$-deformed equipartition theorem for three types Boltzmann factors.

\section{Acknowledgement}

This Work (GNUDFF- 2014-25) was supported by Academy-oriented Research Funds of Development Fund Foundation, Gyeongsang National University, 2014

\section{References:}

[1] C. Beck and E. G. D. Cohen, " Superstatistics," Physica A, 322, 267-275, 2003.
[2] A. G. Bashkirov and A. D. Sukhanov, "The distribution function for a subsystem experiencing temperature fluctuations," J. Exp. Theor. Phys., 95, 440-446, 2002.

[3] C. Tsallis and A. M. C. Souza, "Onstructing a statistical mechanics for Beck-Cohen superstatistics," Phys. Rev. E, 67, 026106,2003 .

[4] C. Beck and E. G. D. Cohen, "Superstatistical generalization of the work fluctuation theorem," Physica A 344, 393-402, 2004.

[5] H. Touchette and C. Beck, "Asymptotics of superstatistics," Phys. Rev. E, 71, 016131, 2005.

[6] C. Beck, E. G. D. Cohen, and H. L. Swinney, “Velocity difference statistics in turbulence," Phys. Rev. E, 72, 056133, 2005.

[7] C. Beck, E. G. D. Cohen, and S. Rizzo, "Atmospheric turbulence and superstatistics," Europhys. News, 36, 189-191, 2005.

[8] C. Vignat, A. Plastino, and A. R. Plastino, "Superstatistics based on the microcanonical ensemble," Nuovo Cimento B, 120, 951-963, 2005.

[9] P.-H. Chavanis, "Coarse-grained distributions and superstatistics," Physica A, 359, 177-212, 2006.

[10] T. Yamano, "Thermodynamical and informational structure of superstatistics," Prog. Theor. Phys. Suppl., 162, 87-96, 2006.

[11] C. Tsallis, "Possible generalization of BoltzmannGibbs statistics,” J. Stat. Phys., 52, 479-487, 1988.

[12] E. Curado and C.Tsallis, "Generalized statistical mechanics: connection with thermodynamics," J. Phys. A, 25, 69-72, 1991.

[13] A. Cho, "A fresh take on disorder, or disorderly science?" Science, 297, 1268-1269, 2002.

[14] L. Nivanen, A. Le Mehaute, Q. A. Wang "Generalized algebra within a nonextensive statistics," Rep. Math. Phys., 52, 437-444, 2003.

[15] E. P. Borges, "A possible deformed algebra and calculus inspired in nonextensive thermostatistics," J. Physica A, 340, 95-101, 2004. 